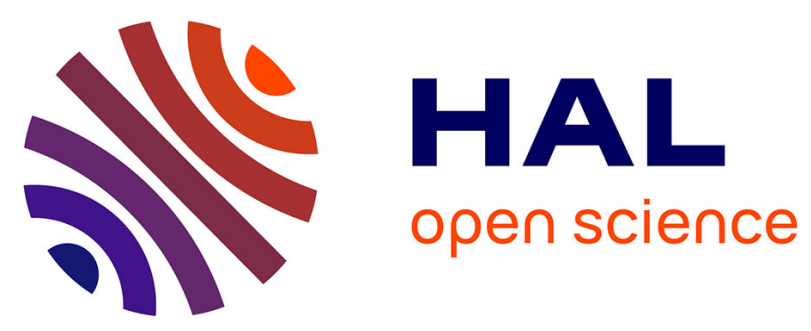

\title{
Clinical Communications Symmetrical drug-related intertriginous and flexural exanthema: A little-known drug allergy
}

Tullia De Risi-Pugliese, Héloïse Barailler, Aurore Hamelin, Emmanuelle Amsler, Hafida Gaouar, Flore Kurihara, Marie Laure Jullie, Eric Dean Merrill, Annick Barbaud, Philippe Moguelet, et al.

\section{To cite this version:}

Tullia De Risi-Pugliese, Héloïse Barailler, Aurore Hamelin, Emmanuelle Amsler, Hafida Gaouar, et al.. Clinical Communications Symmetrical drug-related intertriginous and flexural exanthema: A little-known drug allergy. Journal of Allergy and Clinical Immunology: In Practice, 2020, 8 (9), pp.3185-3189.e4. 10.1016/j.jaip.2020.04.052 . hal-02995700

\section{HAL Id: hal-02995700 \\ https://hal.sorbonne-universite.fr/hal-02995700}

Submitted on 9 Nov 2020

HAL is a multi-disciplinary open access archive for the deposit and dissemination of scientific research documents, whether they are published or not. The documents may come from teaching and research institutions in France or abroad, or from public or private research centers.
L'archive ouverte pluridisciplinaire HAL, est destinée au dépôt et à la diffusion de documents scientifiques de niveau recherche, publiés ou non, émanant des établissements d'enseignement et de recherche français ou étrangers, des laboratoires publics ou privés. 


\section{Symmetrical drug-related intertriginous and flexural exanthema: A little-known drug allergy}

Tullia de Risi-Pugliese, $M D^{a, b}$, Héloïse Barailler, $M^{c}{ }^{c}$, Aurore Hamelin, MD ${ }^{a}$, Emmanuelle Amsler, MD ${ }^{a, b}$, Hafida Gaouar, MD ${ }^{\mathrm{a}}$, Flore Kurihara, $\mathrm{MD}^{\mathrm{a}, \mathrm{b}}$, Marie Laure Jullie, $\mathrm{MD}^{\mathrm{d}}$, Eric Dean Merrill, $\mathrm{MD}^{\mathrm{e}}$, Annick Barbaud, MD, $\mathrm{PhD}^{\mathrm{a}}$,b , Philippe Moguelet, $\mathrm{MD}^{\mathrm{f}}$, Brigitte Milpied-Homsi, $\mathrm{MD}^{\mathrm{b}}$, and

Angèle Soria, $\mathrm{MD}, \mathrm{PhD}^{\mathrm{a}, \mathrm{b}, \mathrm{g}}$

\section{Clinical Implications}

- Symmetrical drug-related intertriginous and flexural exanthema is a flexural eruption that occurs within a few days of drug exposure. Antibiotics and iodinated contrast agents are the most frequent triggers. Skin testing has low sensitivity. Rechallenge identifies the culprit drug without serious manifestations.

The symmetrical drug-related intertriginous and flexural exanthema (SDRIFE) is a delayed flexural exanthema induced by a systemic drug without previous cutaneous sensitization, typically presenting as "a sharply defined symmetrical erythema of the gluteal area and in the flexural or intertriginous folds without systemic symptoms and signs." ${ }^{1}$ SDRIFE clinically resembles Baboon syndrome (BS); however, in BS, patients develop the exanthem after systemic exposure to an allergen to which they have been previously topically sensitized. ${ }^{2}$

We retrospectively reviewed records from 2 French dermatology departments between 2006 and 2018 for adults with SDRIFE as defined by Häusermann et $\mathrm{al}^{1}$ : (1) exposure to systemically administered drug either following the first or subsequent dose, (2) sharply demarcated erythema of the gluteal and/or inguinal area, (3) involvement of at least 1 other flexural localization, (4) symmetry of affected area, and (5) absence of systemic symptoms and signs. Twelve patients were excluded because of previous topical sensitization to the allergen (BS, $\mathrm{n}=2$ ), diffuse maculopapular rash (MPR) with flexural reinforcement $(n=7)$, other causes of flexural rash, for example, acute generalized exanthematous pustulosis (AGEP), toxic erythema due to chemotherapy (TEC) based on clinical and histological findings $(n=2)$, and flexural erythema thought to be drug-induced but without relapse after reexposure $(\mathrm{n}=1)$. Patch tests (PTs) (IQ ultrachambers, Chemotechnique Diagnostics, Velinge, Sweden), skin prick tests (SPTs), and intradermal tests (IDTs) with delayed readings were performed following European guidelines ${ }^{3}$ (Table I). We further reviewed all published cases of SDRIFE since Häusermann et al's publication (2004-2018).

Eighteen patients were included, 10 females and 8 males, with mean and median ages of 57 years (range, 33-83 years). Erythematous patches or plaques affecting a mean of 5 (range,
2-7) large or small skin folds (especially inguinal, gluteal, axillary, and mammary) (Figure 1, Table I) were observed. Four patients had localized skin vesicles or bullae and 1 (no. 16) had mucosal involvement. The eruption occurred shortly after drug exposure (median, 22 hours; mean, 34; range, 0.5-120). The causative drug was discontinued immediately in all patients, 10 were treated with topical steroids, and resolution of lesions was observed within a median of 4 days (mean, 6.2; range, 1-18). Lymphopenia was found in 6 of 12 patients (50\%; range, $350-1400 / \mathrm{mm}^{3}$ ), not explained by a distinct underlying condition.

The most frequent histological pattern, found in 5 of 11 patients who had a skin biopsy, was subepidermal edema with a polymorphous perivascular and interstitial infiltrate of neutrophils, eosinophils, and lymphocytes in the upper dermis. Four patients had a spongiotic and/or lichenoid pattern on skin biopsy and 2 had a nonspecific scant lymphocytic superficial perivascular infiltrate. There was no obvious relationship between histology and the clinical presentation and severity of the rash or timing of skin biopsy.

The suspected drug was an antibiotic in 10 of 18 cases $(56 \%)$, including amoxicillin \pm clavulanate in 4 (22\%), a iodinated contrast agent (ICA) in 5 of 18 (28\%), an analgesic in 2 of 18 , and fluconazole in 1 of 18 (Table I). Fourteen patients had PT, 12 had SPT, and 11 IDT with delayed readings. Testing was performed within 1 month to 9 years of the original reaction, and most patients (13 of 16) were tested within 1 year of the reaction. Positive skin test results were obtained only in 5 patients, all with antibiotics: PT in 3 of 14 cases (21\%): 1 with amoxicillinclavulanate and 2 with pristinamycin; IDT in 2 of 11 cases $(18 \%)$ with amoxicillin \pm clavulanate. Skin test results remained negative in patient number 2 who also had a SDRIFE due to amoxicillin confirmed by rechallenge test (RT). Results of all skin tests performed with ICAs (PT $\mathrm{n}=3$, SPT and IDT $\mathrm{n}=5$ ) were negative. The result of RT with the suspected causative drug, performed in 9 patients with negative skin test results, was always positive, without severe cutaneous or systemic manifestations. There was no obvious relationship between clinical presentation and positivity of skin test results. The median time for SDRIFE recurrence after reexposure to the drug (induced by RT $[n=9]$ or skin tests $[\mathrm{n}=1]$ ) was 12 hours (mean, 15 hours; range 2.5-30), shorter than during the first episode.

SDRIFE is thought to involve a type IV delayedhypersensitivity immune response, because it occurs within a few hours to a few days after drug exposure, and there is evidence for a T-cell-mediated reaction, , 4 though the precise physiopathology is not known. Antibiotics are common triggering agents in the literature $\left(60 \%\right.$ in Häusermann et al's ${ }^{1}$ review, $33 \%$ in our literature review [2004-2018], $\mathrm{n}=51$ [see Tables E1 and E2 in this article's Online Repository at www.jaci-inpractice.org]), especially beta-lactams ( $55 \%$ in Hausermann et al's review and $23 \%$ in our review), but only 5 cases of SDRIFE due to ICAs were reported. ${ }^{5}$ In previously published cases, skin test results were positive more frequently than in our study, $40 \%$ of PTs, $11 \%$ of SPTs, and $70 \%$ of IDTs, for various drugs (antibiotics and others), possibly due to a publication bias. Different methods for identifying the culprit drug might be necessary in SDRIFE. Skin testing on affected sites is one option; however, 
TABLE I. Clinical, biological, and allergological findings

\begin{tabular}{|c|c|c|c|c|c|c|c|c|}
\hline \multirow[b]{2}{*}{ No. } & \multirow[b]{2}{*}{$\begin{array}{l}\text { Sex, } \\
\text { age (y) }\end{array}$} & \multirow[b]{2}{*}{$\begin{array}{l}\text { No. of } \\
\text { occur } \\
\text { rences }\end{array}$} & \multirow{2}{*}{$\begin{array}{l}\text { Delay } \\
\text { between } \\
\text { first drug } \\
\text { intake } \\
\text { and } \\
\text { eruption } \\
\text { (h) }\end{array}$} & \multicolumn{4}{|c|}{ Eruption aspects } & \multirow[b]{2}{*}{$\begin{array}{c}\text { Biological } \\
\text { investigations }\end{array}$} \\
\hline & & & & $\begin{array}{l}\text { Lesions } \\
\text { type }\end{array}$ & $\begin{array}{l}\text { No. of } \\
\text { affected } \\
\text { skin folds }\end{array}$ & $\begin{array}{l}\text { Affected } \\
\text { skin } \\
\text { folds }\end{array}$ & $\begin{array}{l}\text { Other } \\
\text { affected } \\
\text { site(s) }\end{array}$ & \\
\hline$\overline{1}$ & $\mathrm{~F}, 70$ & 1 & 20 & Erythema & 2 & Inguinal, gluteal & $\begin{array}{l}\text { Back (mild } \\
\text { erythema) }\end{array}$ & NA \\
\hline 2 & M, 38 & 2 & 8 & Erythema & 3 & Inguinal, axillary, neck & Back & $\begin{array}{l}\text { CBC: ND, electrolytes, creatininemia, liver enzymes, } \\
\text { CRP: N }\end{array}$ \\
\hline 3 & M, 83 & $\geq 2$ & 48 & Maculopapular erythema & 2 & Inguinal, gluteal & 0 & $\begin{array}{l}\text { CBC: lymphopenia } 1,110 / \mathrm{mm}^{3}(\mathrm{~N}>1,500) \text {, } \\
\text { electrolytes: N, creatininemia: } 642 \mu \mathrm{mol} / \mathrm{L} \dagger \text {, liver } \\
\text { enzymes: N, CRP: } 65 \mathrm{mg} / \mathrm{L} \dagger\end{array}$ \\
\hline 4 & $\mathrm{~F}, 49$ & 2 & 24 & $\begin{array}{l}\text { Maculopapular } \\
\text { erythemaedema } \\
\text { ++vesicles } \\
\text { after second RT }\end{array}$ & 6 & $\begin{array}{l}\text { Inguinal, gluteal, axillary, } \\
\text { neck, mammary, } \\
\text { abdominal }\end{array}$ & 0 & $\begin{array}{l}\text { CBC: mild } \\
\text { neutropenia } 1,390 / \mathrm{mm}^{3} \text {, electrolytes, creatininemia, } \\
\text { CRP: N, liver enzymes: AST 39, ALT } 52(\mathrm{~N}<32) \text {, } \\
\text { GGT } 52(\mathrm{~N}<32) \text {, ALP N, HSV, CMV, EBV } \\
\text { PCR: - }\end{array}$ \\
\hline 5 & $\mathrm{~F}, 50$ & 2 & 30 & Maculopapular erythema & 4 & $\begin{array}{l}\text { Inguinal, popliteal, mammary, } \\
\text { interdigital }\end{array}$ & 0 & $\begin{array}{l}\text { CBC: aggravation of former lymphopenia } 600 / \mathrm{mm}^{3} \text {, } \\
\text { electrolytes: N, creatininemia, liver enzymes: N, } \\
\text { CRP: } 37 \mathrm{mg} / \mathrm{L}, \mathrm{HSV} 1+2, \mathrm{EBV}, \mathrm{CMV}, \text { HHV6 } \\
\text { PCR: - }\end{array}$ \\
\hline 6 & $\mathrm{M}, 81$ & 2 & 20 & Maculopapular erythema & 4 & $\begin{array}{l}\text { Inguinal, axillary, antecubital } \\
\text { fossae, interdigital }\end{array}$ & 0 & $\begin{array}{l}\text { CBC: lymphopenia } 1,020 / \mathrm{mm}^{3} \text {, electrolytes, } \\
\text { creatininemia: N, liver enzymes: GGT } 248, \text { AST } \\
\text { 39, ALT 50, ALP } 112(\mathrm{~N}<35) \dagger \text {, CRP: N, } \\
\text { HSV1+2, EBV, CMV, HHV6 PCR: - }\end{array}$ \\
\hline 7 & M, 68 & 3 & 96 & $\begin{array}{l}\text { Erosive maculopapular } \\
\text { erythema }\end{array}$ & 4 & $\begin{array}{l}\text { Inguinal, gluteal, axillary, } \\
\text { mammary }\end{array}$ & $\begin{array}{l}\text { Outer arms, upper } \\
\text { chest }\end{array}$ & $\begin{array}{l}\text { CBC, electrolytes, creatininemia, liver enzymes: N, } \\
\text { HSV } 1+2, \text { CMV, HHV6 A+B PCR: -, EBV PCR: } \\
3.96 \log , 9,038 \mathrm{UI} / \mathrm{mL}\end{array}$ \\
\hline 8 & $\mathrm{~F}, 33$ & 2 & 10 & Erythema & 5 & $\begin{array}{l}\text { Inguinal, gluteal, axillary, } \\
\text { mammary, antecubital } \\
\text { fossae }\end{array}$ & Lumbar region & $\begin{array}{l}\text { CBC: lymphopenia } 1,210 / \mathrm{mm}^{3} \text {, electrolytes, } \\
\text { creatininemia, liver enzymes, CRP: N }\end{array}$ \\
\hline 9 & M, 77 & 2 & 12 & Erythema & 7 & $\begin{array}{l}\text { Inguinal, gluteal, popliteal, } \\
\text { antecubital fossae, neck, } \\
\text { mammary, abdominal }\end{array}$ & 0 & $\begin{array}{l}\text { CBC: lymphopenia } 720 / \mathrm{mm}^{3} \text {, electrolytes, } \\
\text { creatininemia, liver enzymes, CRP: N, HSV1+2, } \\
\text { CMV, EBV PCR: - }\end{array}$ \\
\hline 10 & M, 36 & 1 & 6 & $\begin{array}{l}\text { Macular erythema, } \\
\text { vesicles }\end{array}$ & 5 & $\begin{array}{l}\text { Inguinal, axillary, antecubital } \\
\text { fossae, interdigital, wrists }\end{array}$ & Dorsum of feet & $\begin{array}{l}\text { CBC: lymphopenia } 1,120 / \mathrm{mm}^{3} \text {, electrolytes, } \\
\text { creatininemia, liver enzymes: N, CRP } 35 \mathrm{mg} / \mathrm{L} \dagger\end{array}$ \\
\hline 11 & M, 65 & 2 & 0.5 & Macular erythema & 4 & $\begin{array}{l}\text { Inguinal, axillary, abdominal, } \\
\text { interdigital }\end{array}$ & 0 & $\begin{array}{l}\text { CBC: lymphopenia } 1,430 / \mathrm{mm}^{3} \dagger \text {, electrolytes, } \\
\text { creatininemia } \mathrm{N} \text {, liver enzymes: ALT } 127(\mathrm{~N}<43 \\
\text { U/L), ALP } 74(\mathrm{~N}<35 \mathrm{U} / \mathrm{L}) \dagger \text {, CRP: } \mathrm{N}\end{array}$ \\
\hline 12 & $\mathrm{~F}, 59$ & 1 & 24 & $\begin{array}{l}\text { Maculopapular erythema } \\
(+2 \text { bullae left hand })\end{array}$ & 6 & $\begin{array}{l}\text { Inguinal, gluteal, axillary, } \\
\text { popliteal, neck, wrists }\end{array}$ & Dorsum of hands & NA \\
\hline 13 & F, 38 & $\geq 2$ & 12 & Erythema & 6 & $\begin{array}{l}\text { Inguinal, gluteal, axillary, } \\
\text { neck, mammary, } \\
\text { abdominal }\end{array}$ & 0 & NA \\
\hline 14 & $\mathrm{~F}, 55$ & 1 & 96 & Erythema & 7 & $\begin{array}{l}\text { Inguinal, gluteal, axillary, } \\
\text { popliteal, antecubital } \\
\text { fossae, neck, abdominal }\end{array}$ & $\begin{array}{l}\text { Anterior thighs and } \\
\text { legs }\end{array}$ & NA \\
\hline 15 & F, 72 & 1 & 48 & $\begin{array}{l}\text { Erythema with purpuric } \\
\text { lesions }\end{array}$ & 6 & $\begin{array}{l}\text { Inguinal, gluteal, axillary, } \\
\text { antecubital fossae, } \\
\text { mammary, abdominal }\end{array}$ & Inner arms, sides & $\begin{array}{l}\text { CBC: elevated PMN } 15,830 / \mathrm{mm}^{3}(\mathrm{~N}<8,000) \dagger, \\
\text { lymphopenia } 350 / \mathrm{mm}^{3}+\text {, electrolyltes, } \\
\text { creatininemia, liver enzymes: N, CRP: } 74.2 \dagger \mathrm{mg} / \mathrm{L}\end{array}$ \\
\hline 16 & $\mathrm{~F}, 43$ & 1 & 24 & $\begin{array}{l}\text { Macular erythema, } \\
\text { vesicles }\end{array}$ & 7 & $\begin{array}{l}\text { Inguinal, gluteal, axillary, } \\
\text { mammary, abdominal, } \\
\text { interdigital, nasolabial }\end{array}$ & $\begin{array}{l}\text { Vesicles of the soft } \\
\text { palate }\end{array}$ & $\begin{array}{l}\text { CBC: hyperleucocytosis } 13,000 / \mathrm{mm}^{3} \dagger \text {, electrolytes, } \\
\text { creatininemia: N, liver enzymes: ALT } 50 \mathrm{UI} / \mathrm{L}, \\
\text { AST } 29 \mathrm{UI} / \mathrm{L} \text {, ALP } 67 \mathrm{UI} / \mathrm{L} \neq \text {, CRP: } 100 \mathrm{mg} / \mathrm{L} \dagger\end{array}$ \\
\hline 17 & M, 69 & 1 & 12 & $\begin{array}{l}\text { Erythema, secondarily } \\
\text { bleeding erosions and } \\
\text { scaling }\end{array}$ & 5 & $\begin{array}{l}\text { Inguinal, gluteal, axillary, } \\
\text { neck, interdigital }\end{array}$ & Feet & $\begin{array}{l}\text { CBC: elevated PMN } 11,670 / \mathrm{mm}^{3} \dagger \text {, electrolytes, } \\
\text { creatininemia, liver enzymes: N, CRP: } 156 \mathrm{mg} / \mathrm{L} \dagger\end{array}$ \\
\hline 18 & $\mathrm{~F}, 41$ & 1 & 120 & Erythema & 5 & $\begin{array}{l}\text { Inguinal, gluteal, axillary, } \\
\text { mammary }\end{array}$ & 0 & NA \\
\hline
\end{tabular}

$A L P$, Alkaline phosphatase; $A L T$, aspartate aminotransferase; $A M O X$, amoxicillin; $A M O X-C L A V$, amoxicillin-clavulanate; $A S T$, alanine aminotransferase; $C B C$, complete blood cell count; Chem, Chemotechnique; $C L I N D A$, clindamycin; $C M V$, cytomegalovirus; $C R P$, C-reactive protein; $C S$, corticosteroid; $E B V$, Epstein-Barr virus; $F$, female; $G G T$, gamma-glutamyl transferase; $H 1$, antihistaminic H1; HHV6, human herpesvirus $6 ; H S V$, herpes simplex virus; $M$, male; $N$, normal; $N A$, data not available; $N D$, not done; $P B 19$, parvovirus B19; $P C R$, polymerase chain reaction; $P M N$, polymorphonuclear neutrophil; $P R I S T$, pristinamycin; + , positive; - , negative; $R T+$, recurrence of SDRIFE; $R T-$, no recurrence of SDRIFE; SULFTX, sulfamethoxazole; TS, topical steroid.

Note: For patient numbers 9 and 13: RTs were performed with alternative $\beta$-lactams (ceftriaxone, cefpodoxime) after confirmed hypersensitivity to amoxicillin \pm clavulanate, and results were negative.

*Most drug PTs were performed using commercialized preparations (Chemotechnique Diagnostic, Vellinge, Sweden). If not available, the hospital pharmacy freshly prepared the drug PT at nonirritant concentrations as previously recommended in the literature.

$\dagger$ Abnormally former to the SDRIFE, explained by underlying condition (eg, infection).

$\ddagger$ Abnormally possibly explained by underlying condition, or by the SDRIFE (unknown). 


\begin{tabular}{|c|c|c|c|c|c|c|c|}
\hline Treatment & $\begin{array}{c}\text { Time to } \\
\text { healing (d) }\end{array}$ & $\begin{array}{l}\text { Suspected } \\
\text { drugs }\end{array}$ & PTs* & Prick tests & IDTs & $\begin{array}{l}\text { RT result } \\
\text { and delay } \\
\text { for SDRIFE } \\
\text { recurrence (h) }\end{array}$ & $\begin{array}{l}\text { Drug } \\
\text { confirmed } \\
\text { by tests or } \\
\text { rechallenge }\end{array}$ \\
\hline TS & NA & PRIST & PRIST (Chem) - & PRIST - & ND & $\begin{array}{l}\text { PRIST }(1 \mathrm{~g})+,<24 \text {, with } \\
\text { erythematous reaction on } \\
\text { site of previous SPT }\end{array}$ & PRIST \\
\hline TS & NA & AMOX & AMOX (Chem): - & $\begin{array}{l}\text { AMOX, cefpodoxim, } \\
\text { ceftriaxone } \\
\text { (undiluted): - }\end{array}$ & $\begin{array}{l}\text { AMOX, ceftriaxone } \\
\left.\quad \text { (diluted to } 10^{-1}\right) \text { : - }\end{array}$ & $\operatorname{AMOX}(1 \mathrm{~g})+, 12$ & AMOX \\
\hline TS & 15 & $\begin{array}{l}\text { Oxycodone, oxycodone } \\
\text { chloryhdrate }\end{array}$ & ND & ND & ND & ND & - \\
\hline TS & NA & Iodixanol & $\begin{array}{l}\text { Iodixanol, iomeprol, iohexol, } \\
\quad \text { ioxitalamate (undiluted): - }\end{array}$ & $\begin{array}{l}\text { Iodixanol, iomeprol, } \\
\quad \text { iohexol, ioxitalamate } \\
\text { (undiluted): - }\end{array}$ & $\begin{array}{l}\text { Iodixanol, iomeprol, } \\
\quad \text { iohexol, ioxitalamate } \\
\text { (diluted to } 10^{-1} \text { ): - }\end{array}$ & $\begin{array}{l}\text { Iodixanol }(50 \mathrm{~mL})+, 2.5 \\
\text { Iomeprol }(50 \mathrm{~mL}):+, 2.5 \\
\quad \text { (cross-reactivity) }\end{array}$ & Iodixanol \\
\hline TS & 4 & $\begin{array}{l}\text { Ioversol ( } 3 \text { episodes with } \\
\quad \text { Ioversol) }\end{array}$ & $\begin{array}{l}\text { Ioversol, iohexol, iomeprol, } \\
\text { iodixanol ioxitalamate, } \\
\text { iobitridol (undiluted): - }\end{array}$ & $\begin{array}{l}\text { Ioversol, iohexol, } \\
\text { iomeprol, iodixanol } \\
\text { ioxitalamate, } \\
\text { iobitridol } \\
\text { (undiluted): - }\end{array}$ & $\begin{array}{l}\text { Ioversol, iohexol, } \\
\text { iomeprol, iodixanol } \\
\text { ioxitalamate, } \\
\text { iobitridol (diluted to } \\
10^{-1} \text { ): - }\end{array}$ & $\begin{array}{l}\text { Iomeprol }(50 \mathrm{~mL})+, 24 \\
\quad \text { (cross-reactivity) } \\
\text { Iodixanol }(50 \mathrm{~mL})+, 24 \\
\quad \text { (cross-reactivity) } \\
\text { Iobitridol }(50 \mathrm{mLl}):-\end{array}$ & ICAs (group A) \\
\hline TS & 2 & Iomeprol & ND & $\begin{array}{l}\text { Iomeprol, iobitridol, } \\
\text { iodixanol } \\
\quad \text { (undiluted): - }\end{array}$ & $\begin{array}{l}\text { Iomeprol, iobitridol, } \\
\text { Iodixanol (diluted to } \\
10^{-1} \text { ): - }\end{array}$ & $\begin{array}{l}\text { Iomeprol }(50 \mathrm{~mL})+, 12 \\
\text { Iobitridol }(50 \mathrm{~mL})-\end{array}$ & Iomeprol \\
\hline TS & NA & $\begin{array}{l}\text { Iomeprol (for both } \\
\text { episodes) + ibuprofene, } \\
\text { lamaline (first episode), } \\
\text { terbinafine (second } \\
\text { episode) }\end{array}$ & ND & $\begin{array}{l}\text { Iomeprol, iodixanol, } \\
\text { iohexol, iompromide } \\
\text { (undiluted): - }\end{array}$ & $\begin{array}{l}\text { Iomeprol, iodixanol, } \\
\text { iohexol, } \\
\text { iompromide (diluted } \\
\text { to } 10^{-1} \text { ): - }\end{array}$ & Iomeprol $(50 \mathrm{~mL})+, 24$ & Iomeprol \\
\hline NA & 3 & $\begin{array}{l}\text { CLINDA, nefopam, oxytocin, } \\
\text { esomeprazole, ketoprofen, } \\
\text { levobupicaine, sufentanil, } \\
\text { tranexamic acid }\end{array}$ & $\begin{array}{l}\text { CLINDA (Chem): - nefopam, } \\
\text { oxytocin, esomeprazol, } \\
\text { eketoprofen, levobupicain, } \\
\text { esufentanil, tranexamic acid } \\
\text { (30\% in water): - }\end{array}$ & $\begin{array}{l}\text { CLINDA (Chem), } \\
\text { nefopam, oxytocin, } \\
\text { esomeprazole, } \\
\text { ketoprofen, } \\
\text { levobupicaine, } \\
\text { sufentanil, } \\
\text { tranexamic acid } \\
\text { (undiluted): - }\end{array}$ & $\begin{array}{l}\text { CLINDA, nefopam, } \\
\text { oxytocin, } \\
\text { esomeprazole, } \\
\text { ketoprofen, } \\
\text { levobupicaine, } \\
\text { sufentanil, } \\
\text { tranexamic acid } \\
\text { (diluted to } \\
\left(0^{-1}\right):-\end{array}$ & CLINDA $(300 \mathrm{mg})+, 3$ & CLINDA \\
\hline NA & 10 & AMOX & AMOX (Chem): - & $\begin{array}{l}\text { AMOX, ceftriaxone, } \\
\text { cefpodoxime } \\
\text { (undiluted):- }\end{array}$ & $\begin{array}{l}\text { AMOX (diluted to } \\
10^{-1} \text { ): + with SDRIFE } \\
\text { recurrence } 30 \mathrm{~h} \text { after } \\
\text { IDT; ceftriaxone } \\
\text { (diluted to } 10^{-1} \text { ): - }\end{array}$ & $\begin{array}{l}\text { Ceftriaxone }(1 \mathrm{~g}),- \\
\quad \text { Cefpodoxime }(100 \mathrm{mg}):-\end{array}$ & AMOX \\
\hline TS & 4 & Paracetamol, tramadol & ND & ND & ND & ND & - \\
\hline TS, H1 & 1 & Ioxaglate & $\begin{array}{l}\text { Ioxaglate, iodixanol, iomeprol, } \\
\text { iopamidol, iopromide } \\
\text { (undiluted): - }\end{array}$ & $\begin{array}{l}\text { Ioxaglate, iodixanol, } \\
\text { iomeprol, iopamidol, } \\
\text { iopromide } \\
\text { (undiluted): - }\end{array}$ & $\begin{array}{l}\text { Ioxaglate iodixanol, } \\
\text { iomeprol, iopamidol, } \\
\text { iopromide (diluted to } \\
10^{-1} \text { ): - }\end{array}$ & $\begin{array}{l}\text { Ioxaglate } 50 \mathrm{~mL}+, 6 \\
\quad \text { Iodixanol - }\end{array}$ & Ioxaglate \\
\hline TS & 5 & SULFTX & SULFTX (Chem): - & SULFTX (undiluted): - & $\begin{array}{l}\text { SULFTX (diluted to } \\
\left.10^{-1}\right):-/-\end{array}$ & ND & - \\
\hline $\mathrm{H} 1$ & 2 & AMOX-CLAV & AMOX (Chem): - & $\begin{array}{l}\text { AMOX-CLAV, } \\
\text { ceftriaxone, } \\
\text { cefpodoxime } \\
\text { (undiluted):- }\end{array}$ & $\begin{array}{l}\text { AMOX-CLAV (diluted } \\
\text { to } 10^{-1} \text { ): }- \text { and }+ \\
\text { when retested after } \\
\text { positive RT result) } \\
\text { Ceftriaxone (diluted } \\
\text { to } 10^{-1} \text { ): }-\end{array}$ & $\begin{array}{l}\text { AMOX-CLAV }(1 \mathrm{~g}):+, 12 \\
\text { Ceftriaxone }(1 \mathrm{~g}):- \\
\quad \text { Cefpodoxime }(100 \mathrm{mg}):-\end{array}$ & AMOX-CLAV \\
\hline NA & NA & $\begin{array}{l}\text { AMOX-CLAV, ofloxacin, } \\
\text { tramadol }\end{array}$ & $\begin{array}{l}\text { AMOX-CLAV ( } 30 \% \text { pet.): }+ \text {, } \\
\text { ofloxacin: - }\end{array}$ & ND & ND & ND & AMOX-CLAV \\
\hline H1 & 18 & PRIST & $\begin{array}{l}\text { PRIST (10\% pet.): }+ \\
\quad \text { Pristinamycin ( } 30 \% \text { water): }-\end{array}$ & ND & ND & ND & PRIST \\
\hline \multirow[t]{2}{*}{ TS } & 28 & Vancomycin & $\begin{array}{l}\text { Vancocmycin ( } 30 \% \text { pet., } 30 \% \\
\text { water): - }\end{array}$ & $\begin{array}{l}\text { Vancomycin (undiluted): } \\
\quad-\end{array}$ & $\begin{array}{l}\text { Vancomycin (diluted to } \\
10^{-3} \text { ): irritative at } \\
20 \mathrm{~min},- \text { at D2 }\end{array}$ & ND & - \\
\hline & 35 & PRIST, ofloxacin & $\begin{array}{l}\text { PRIST (10\% pet.): +, PRIST ( } 30 \% \\
\text { water): +, erythromycin, } \\
\text { spiramycin, clarithromycin, } \\
\text { norfloxacin, ciprofloxacin, } \\
\text { acetaminophen (10\% pet.): - }\end{array}$ & ND & ND & ND & PRIST \\
\hline NA & 42 & Fluconazole & $\begin{array}{l}\text { Fluconazole ( } 30 \% \text { pet.), econazole } \\
\text { cream (undiluted): - }\end{array}$ & ND & ND & ND & - \\
\hline
\end{tabular}




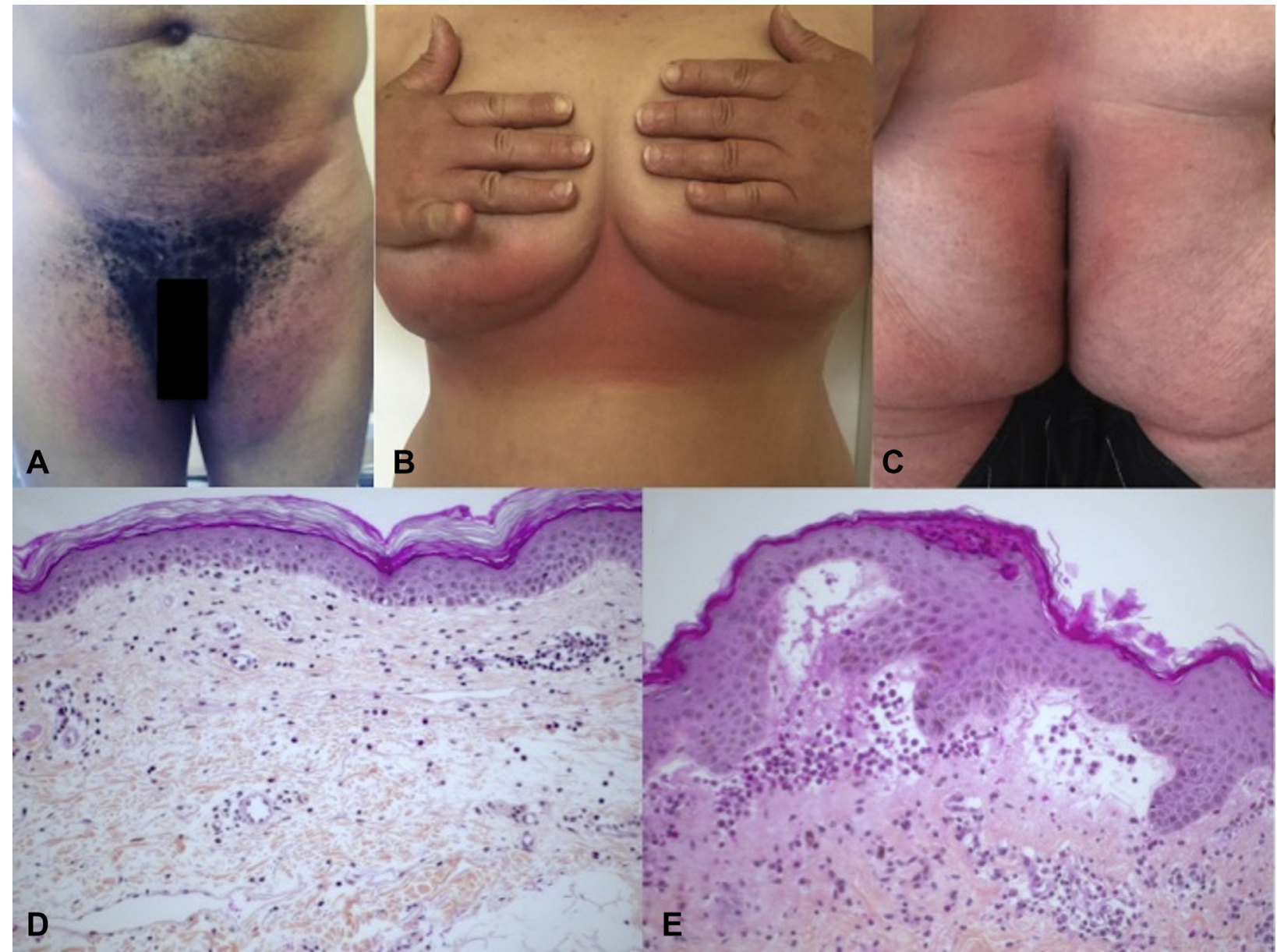

FIGURE 1. SDRIFE. (A-C) Clinical aspects. Maculopapular, sharply demarcated erythema of the $(A)$ inguinal (patient no. 10), $(B)$ mammary (patient no. 5), and (A) gluteal folds (patient no. 9). (D and E) Histology on skin biopsy (most frequently found pattern). Subepidermal edema, perivascular and interstitial infiltrate of neutrophils, eosinophils, and lymphocytes in the upper dermis with minimal leucocytoclasia (patient nos. 4 and 10).

site-specific skin testing with PT and/or IDT has been negative in 3 cases to date. ${ }^{4,6,7}$ Thus, RT, result for which was positive in 9 of 9 cases in our study and 13 of 13 in our literature review (2004-2018), appears to be the most reliable way to identify the culprit drug, without serious manifestations during recurrence.

SDRIFE is a syndrome characterized by a drug-induced flexural eruption, but it cannot be clearly defined on the basis of histology and skin testing. We observed that using Häusermann et al's 5 diagnostic criteria may have led to misdiagnosis: we excluded 2 patients who fulfilled these criteria but where alternate diagnoses of TEC in one case and TEC with features of AGEP in the other were better fits. Typical SDRIFE occurring within a few days after drug exposure can be clinically diagnosed. However, in patients with pustules, bullae, erosions, and atypical chronology, or in patients receiving chemotherapy, a skin biopsy should be performed to rule out another diagnosis. Moreover, the well-demarcated erythema of the folds is an important clinical aspect for diagnosis, to distinguish an MPR that can have flexural reinforcement. Taken together, we suggest the addition of a sixth criterion: (6) exclusion of another cause of flexural eruption (including AGEP, TEC, psoriasis, fixed drug eruption, MPR with flexural reinforcement, systemic contact dermatitis), with skin biopsy in cases with an atypical clinical presentation.

${ }^{a}$ AP-HP, Service de Dermatologie et Allergologie, Hôpital Tenon, Paris, France ${ }^{\mathrm{b}}$ Sorbonne Université, UPMC University, Paris, France

${ }^{\mathrm{c} S}$ Service de Dermatologie, Hôpital Saint André, Bordeaux, France

${ }^{\mathrm{d}}$ Service d'Anatomopathologie, Hôpital Haut Lévèque, Bordeaux, France

e Department of Dermatology, University of California San Francisco, San Francisco, Calif

fAP-HP, Service d'Anatomopathologie, Hôpital Tenon, Paris, France

${ }^{\mathrm{g}}$ Inserm 1135, Centre d'Immunologie et des Maladies Infectieuses (Cimi-Paris), Paris, France

Conflicts of interest: The authors declare that they have no relevant conflicts of interest.

Received for publication August 23, 2019; revised April 8, 2020; accepted for publication April 21, 2020.

Available online May 4, 2020.

Corresponding author: Tullia de Risi-Pugliese, MD, Service de Dermatologie et Allergologie, Hôpital Tenon, 4 rue de la Chine, 75020 Paris, France. E-mail: tullia. derisi@gmail.com.

2213-2198

C 2020 American Academy of Allergy, Asthma \& Immunology

https://doi.org/10.1016/j.jaip.2020.04.052 


\section{REFERENCES}

1. Häusermann P, Harr T, Bircher AJ. Baboon syndrome resulting from systemic drugs: is there strife between SDRIFE and allergic contact dermatitis syndrome? Contact Dermatitis 2004;51:297-310.

2. Miyahara A, Kawashima H, Okubo Y, Hoshika A. A new proposal for a clinicaloriented subclassification of baboon syndrome and a review of baboon syndrome. Asian Pac J Allergy Immunol 2011;29:150-60.

3. Barbaud A, Gonçalo M, Bruynzeel D, Bircher A, European Society of Contact Dermatitis. Guidelines for performing skin tests with drugs in the investigation of cutaneous adverse drug reactions. Contact Dermatitis 2001; 45:321-8.
4. Barbaud A, Tréchot P, Granel F, Lonchamp P, Faure G, Schmutz JL, et al. A baboon syndrome induced by intravenous human immunoglobulins: report of a case and immunological analysis. Dermatol Basel Switz 1999;199:258-60.

5. Tasker F, Fleming H, McNeill G, Creamer D, Walsh S. Contrast media and cutaneous reactions, part 2: delayed hypersensitivity reactions to iodinated contrast media. Clin Exp Dermatol 2019;44:844-60.

6. Ozkaya E, Babuna G. A challenging case: symmetrical drug related intertriginous and flexural exanthem, fixed drug eruption, or both? Pediatr Dermatol 2011;28:711-4.

7. Ferreira O, Mota A, Morais P, Cunha AP, Azevedo F. Symmetrical drugrelated intertriginous and flexural exanthema (SDRIFE) induced by telmisartan-hydrochlorothiazide. Cutan Ocul Toxicol 2010;29:293-5. 


\section{ONLINE REPOSITORY}

TABLE E1. Comparison between present study, literature review, and the Häusermann et al ${ }^{\mathrm{E} 1}$ review (2004)

\begin{tabular}{|c|c|c|c|c|c|c|c|c|c|c|c|c|c|c|}
\hline & \multirow[b]{2}{*}{$\mathrm{n}$} & \multirow[b]{2}{*}{$\begin{array}{c}\text { Age }(y), \\
\text { mean } \\
\text { (median) }\end{array}$} & \multirow[b]{2}{*}{$\begin{array}{c}\text { Sex: } \\
\text { M/F, n }\end{array}$} & \multirow{2}{*}{$\begin{array}{l}\text { Delay between } \\
\text { first drug intake } \\
\text { and eruption (h), } \\
\text { median (mean) }\end{array}$} & \multicolumn{6}{|c|}{ Culprit drug, $\mathrm{n}(\%)$} & \multicolumn{4}{|c|}{ Allergological workup, $\mathrm{n}(\%)$} \\
\hline & & & & & ATB, $n(\%)$ & $\beta$-Lactam & Amoxicillin & $\begin{array}{c}\text { lodinated } \\
\text { contrast } \\
\text { media }\end{array}$ & $\begin{array}{l}\text { Chemotherapy or } \\
\text { immunotherapy }\end{array}$ & Others & $\begin{array}{l}\text { Positive } \\
\text { PT/PT } \\
\text { performed }\end{array}$ & $\begin{array}{c}\text { Positive } \\
\text { SPT/SPT } \\
\text { performed }\end{array}$ & $\begin{array}{l}\text { Positive } \\
\text { IDT/IDT } \\
\text { performed }\end{array}$ & $\begin{array}{c}\text { Positive } \\
\text { RT/RT } \\
\text { performed }\end{array}$ \\
\hline $\begin{array}{c}\text { Häusermann et al, } \\
2004 \text { (review }+ \\
\text { original cases) }\end{array}$ & $40+2$ & $48(55)$ & $30 / 12$ & $48(72)$ & $25(60)$ & $22(52)$ & $14(33)$ & $0(0)$ & $3(12)$ & $14(64)$ & 12 of $24(50)$ & 2 of $10(20)$ & 3 of $5(60)$ & 4 of $4(100)$ \\
\hline $\begin{array}{l}\text { Literature review } \\
\quad(2004-2018)\end{array}$ & 51 & $46(53)$ & $27 / 24$ & $48(285)$ & $17(33)$ & $10(20)$ & $5(10)$ & $5(10)$ & $2(4)$ & $27(53)^{*}$ & 7 of $24(29) \dagger$ & 0 of $8(0)$ & 4 of $5(80) \ddagger$ & 13 of $13(100)$ \\
\hline Present study & 18 & $57(57)$ & $10 / 8$ & $22(34)$ & $10(56)$ & $4(22)$ & $4(22)$ & $5(28)$ & $0(0)$ & $2(11)$ & 3 of $14(21)$ & 0 of $12(0)$ & 2 of 11 (18) & 9 of $9(100)$ \\
\hline
\end{tabular}

\section{$F$, Female; $M$, male.}

*Other drugs included itraconazole, thiamine disulphide, golimumab, terbinafine, zoledronic acid, etoricoxib, everolimus, codeine, infliximab, paracetamol, ranitidine, coix lacryma jobi, celecoxib, hydroxyzine, omeprazole, etonorgestrel,

clozapine, rivastigmine, telmisartan-hydrochlorothiazide, risperidone, valacyclovir, palifermin (keratinocyte growth factor), prednisolone, and methyprednisolone.

$\dagger$ Positive PT result obtained with clarithromycine, etoricoxib, clindamycin, hydroxyzine, etonorgestrel, and Iomeprol prednisolone

†Positive IDT result obtained with bortezomib, clindamycin, iomeprol, and methylprednisolone.

thes 
TABLE E2. Published cases of SDRIFE (literature review 2004-2018): Culprit drug and results of allergological workup

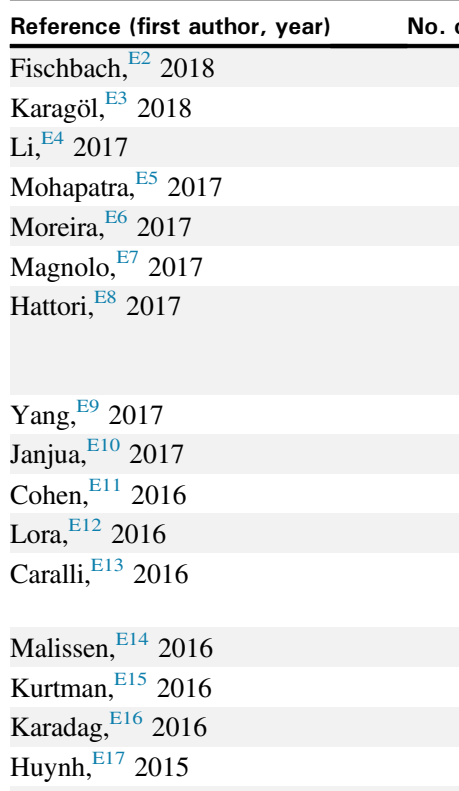

Culprit drug

Allergological workup

of cases

Ceftriaxone/daptomycine/clindamycin

Ampicillin-sulbactam IV

Doxycycline

Itraconazole

Clarithromcyin

Cefuroxime

Thiamine disulfide

-

1

(n)

1

PT: Negative

PT: Positive

-

PT: Negative

SPT: Negative

RT: Positive

Golimumab

Terbinafine

Zoledronic acid

Ceftazidime

Etoricoxib$$
\text { - }
$$

PT: Negative

Bortezomib

Everolimus

Amoxiclline

ICA

ICA

ICA

Clindamycin

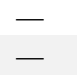

PT: Positive

RT: Positive

IDT: Positive

-

PT: Negative

Morales-Cabeza, ${ }^{\text {E18 }} 2015$

1

Codeine

-

-

PT: Positive

IDT: Positive

Erfan, ${ }^{\text {E19 }} 2015$

1

PT: Negative

SPT: Negative

RT: Positive

Bulur, ${ }^{\text {E20 }} 201$

Infliximab

Obara, ${ }^{\text {E21 }} 2014$

Paracetamol = acetaminophen

RT: Positive

Binitha, ${ }^{\text {E22 }} 2014$

Ranitidine

Sikar Akturk, ${ }^{\text {E23 }} 2014$

Metronidazole

Choi, ${ }^{\text {E24 }} 2014$

Can, ${ }^{\mathrm{E} 25} 2014$

Coix lacryma jobi

Kim, ${ }^{\text {E26 }} 2014$

Cefixime and clarithromycin

Celecoxib

PT: Negative

RT: Positive

PT: Negative

Blackmur, ${ }^{\text {E27 }} 2013$

Benzyl penicillin

Hydroxyzine

-

PT: Negative

PT: Negative

RT: Positive

Akkari, ${ }^{\mathrm{E} 28} 2013$

1

Lee, ${ }^{\text {E29 }} 2013$

1

Culav, ${ }^{\text {E30 }} 2013$

1

Lugovic-Mihic, ${ }^{\text {E31 }} 2013$

Dogru, ${ }^{\text {E32 }} 2012$

1

1

Kardaun, ${ }^{\mathrm{E} 33} 2012$

2

Peeters, ${ }^{\text {E34 }} 2012$

Rao, ${ }^{\text {E35 }} 2012$

1

2

Ozkaya, ${ }^{\text {E36 }} 2011$

1

Allain-Veyrac, ${ }^{\text {E37 }} 2011$

Chong, ${ }^{\text {E38 }} 2010$

Ferreira, ${ }^{\mathrm{E} 39} 2010$

Elmariah, ${ }^{\mathrm{E} 40} 2009$

1

1

1

1

illin/tazobactam/phenytoin

Sulfamethoxazole-trimethoprim

Paracetamol

Amoxicillin-clavulanate

Omeprazole

Omeprazole

Nuvaring: etonorgestrel

Clozapine

Clozapine

Amoxicillin

Rivastigmine

Cloxacillin

Telmisartan-hydrochlorothiazide

CR011-vcMMAE expermimental mAb-Auristatin E conjugate

$$
\text { - }
$$

PT: Positive

RT: Positive

PT: Negative

SPT: Negative

PT: Negative

SPT: Negative

PT: Negative

-

PT: Positive

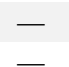

PT: Negative

RT: Positive

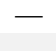

PT: Negative

RT: Positive 
TABLE E2. (Continued)

Reference (first author, year)

No. of cases

Akay, ${ }^{\mathrm{E} 41} 2009$

Thierman, ${ }^{\mathrm{E} 42} 2009$

Daito, ${ }^{\mathrm{E} 43} 2009$

Handisurya, ${ }^{\mathrm{E} 44} 2009$

1
Culprit drug

Risperidone

ICA

Valacyclovir

Amoxicillin/sulbactam

Diaz Ley, ${ }^{\mathrm{E} 45} 2008$

Dhingra, ${ }^{\text {E46 }} 2007$

Arnold, ${ }^{\text {E47 }} 2007$

Treudler, ${ }^{\mathrm{E} 48} 2006$
1

1

1
Palifermin (keratinocyte growth factor)
Cefadroxil/paracétamol/cough-mixture for acute respiratory infection Iomeprol

Allergological workup

PT: Negative

RT: Positive

PT: Negative

RT: Positive

PT: Negative

SPT: Negative

IDT: Negative

RT: Positive

$$
\text { - }
$$

PT: Positive

SPT: Negative

IDT: Positive

RT: Positive

PT: Positive

SPT: Negative 


\section{REFERENCES}

E1. Häusermann P, Harr T, Bircher AJ. Baboon syndrome resulting from systemic drugs: is there strife between SDRIFE and allergic contact dermatitis syndrome? Contact Dermatitis 2004;51:297-310.

E2. Fischbach S, Hall N, Bains S, Selvaraj V. An unusual case of worsening cellulitis: baboon syndrome or SDRIFE. BMJ Case Rep 2018;2018: bcr2018224503.

E3. Karagöl C, Ceran A, Güngör A, Akman AÖ, Misirlioğlu ED. Baboon syndrome associated with ampicillin sulbactam. J Allergy Clin Immunol Pract 2018;6:2106-7.

E4. Li DG, Thomas C, Weintraub GS, Mostaghimi A. Symmetrical drug-related intertriginous and flexural exanthema induced by doxycycline. Cureus 2017;9: e1836.

E5. Mohapatra M, Panda M, Kar BR, Raj C. Symmetric drug-related intertriginous and flexural exanthema due to itraconazole: an uncommon side effect of a commonly used drug. Indian Dermatol Online J 2017:8:501-3.

E6. Moreira C, Cruz MJ, Cunha AP, Azevedo F. Symmetrical drug-related intertriginous and flexural exanthema induced by clarithromycin. An Bras Dermatol 2017;92:587-8.

E7. Magnolo N, Metze D, Ständer S. Pustulobullöse Variante eines SDRIFE (symmetrical drug-related intertriginous and flexural exanthema). J Dtsch Dermatol Ges J Ger Soc Dermatol JDDG 2017;15:657-9.

E8. Hattori Y, Matsuyama K, Shu E, Kanoh H, Seishima M. Symmetrical drugrelated intertriginous and flexural exanthema possibly due to thiamine disulfide. J Dermatol 2017;44:e115-6.

E9. Yang S-Y, Lan C-C, Hu SC-S. Symmetrical drug-related intertriginous and flexural exanthema (SDRIFE) induced by golimumab. Int J Dermatol 2017;56: $571-2$.

E10. Janjua SA, Pastar Z, Iftikhar N, Ammad S. Intertriginous eruption induced by terbinafine: a review of baboon syndrome. Int J Dermatol 2017;56:100-3.

E11. Cohen PR. Zoledronic acid-associated symmetrical drug-related intertriginous and flexural exanthema (SDRIFE): report of baboon syndrome in a woman with recurrent metastatic breast cancer after receiving zoledronic acid. Dermatol Online 2015;21:13030.

E12. Lora V, Capitanio B, Cota C. A symmetrical flexural rash. Pediatr Dermatol 2016;33:345-6.

E13. Caralli ME, Seoane Rodríguez M, Rojas Pérez-Ezquerra P, Pelta Fernández R, De Barrio Fernández M. Symmetrical drug-related intertriginous and flexural exanthema (SDRIFE) caused by etoricoxib. J Investig Allergol Clin Immunol 2016;26:128-9.

E14. Malissen N, Bourrain J-L, Chiriac A, Vincent L, Dereure O, Du-Thanh A, et al. Symmetrical intertriginous and flexural exanthema due to bortezomib (a proteasome inhibitor) given for myeloma. Acta Derm Venereol 2016;96:995-6.

E15. Kurtzman DJB, Oulton J, Erickson C, Curiel-Lewandrowski C. Everolimusinduced symmetrical drug-related intertriginous and flexural exanthema (SDRIFE). Dermat Contact Atopic Occup Drug 2016;27:76-7.

E16. Karadag AS, Ozlu E, Akdeniz N, Uzuncakmak TK, Turkoglu Z, Ozkanli S, et al. Oral mucosal involvement and petechial lesions: a SDRIFE case with unusual findings. Cutan Ocul Toxicol 2016;35:157-9.

E17. Huynh T, Hughey LC, McKay K, Carney C, Sami N. Systemic drug-related intertriginous and flexural exanthema from radio contrast media: A series of 3 cases. JAAD Case Rep 2015;1:147-9.

E18. Morales-Cabeza C, Caralli Bonett ME, Micozzi S, Seoane Rodríguez M, Rojas-Pérez-Ezquerra P, de Barrio Fernández M. SDRIFE-like reaction induced by an intradermal skin test with clindamycin: A case report. J Allergy Clin Immunol Pract 2015;3:976-7.

E19. Erfan G, Yanik ME, Kaya S, Oznur M, Kulac M. Symmetrical drug-related intertriginous and flexural exanthema due to codeine. Indian J Dermatol Venereol Leprol 2015;81:405-6.

E20. Bulur I, Keseroglu HO, Saracoglu ZN, Gönül M. Symmetrical drug-related intertriginous and flexural exanthema (baboon syndrome) associated with infliximab. J Dermatol Case Rep 2015;9:12-4.

E21. Obara K, Maejima H, Katayama C, Takasu H, Amoh Y. A case of symmetrical drug related intertriginous and flexural exanthema induced by acetaminophen. J Dermatol 2014;41:1132-3.

E22. Binitha MP, Sasidharanpillai S, John R, Sherjeena PVB. Symmetrical drugrelated intertriginous and flexural exanthema due to ranitidine. Indian J Pharmacol 2014;46:551-2.
E23. Şikar Aktürk A, Bayramgürler D, Salman S, Yıldız KD, Odyakmaz Demirsoy E. Symmetrical drug-related intertriginous and flexural exanthema (SDRIFE) induced by oral metronidazole. Cutan Ocul Toxicol 2014;33:337-8.

E24. Choi MJ, Oh SH, Cho SB. Coix lacryma-jobi associated symmetrical drugrelated intertriginous and flexural exanthema. Int J Dermatol 2014;53:e301-3.

E25. Can C, Yazicioglu M, Ozdemir PG, Kilavuz S4, Tastekin E. Symmetrical drugrelated intertriginous and flexural exanthema induced by two different antibiotics. Allergol Immunopathol (Madr) 2014;42:173-5.

E26. Kim BJ, Kim HS, Lee JY, Kim HO, Park YM, La HO. Symmetrical drugrelated intertriginous and flexural exanthema caused by celecoxib. Int J Dermatol 2014;53:e1-3.

E27. Blackmur JP, Lammy S, Baring DEC. Baboon syndrome: an unusual complication arising from antibiotic treatment of tonsillitis and review of the literature. BMJ Case Rep 2013;2013:bcr2013201977.

E28. Akkari H, Belhadjali H, Youssef M, Mokni S, Zili J. Baboon syndrome induced by hydroxyzine. Indian J Dermatol 2013;58:244.

E29. Lee HY, Philippidou M, Schey S, Selway R, Walsh S, Creamer D. Flexural eruption in two hospitalized patients. Clin Exp Dermatol 2013;38:943-5.

E30. Culav I, Ljubojevic S, Buzina DS. Baboon syndrome/SDRIFE due to sulfamethoxazole-trimethoprim. Int J Dermatol 2013;52:1159-60.

E31. Lugović-Mihić L, Duvančić T, Vučić M, Situm M, Kolić M, Mihić J. SDRIFE (baboon syndrome) due to paracetamol: case report. Acta Dermatovenerol Croat ADC 2013;21:113-7.

E32. Dogru M, Ozmen S, Ginis T, Duman H, Bostanci I. Symmetrical drug-related intertriginous and flexural exanthema (baboon syndrome) induced by amoxicillin-clavulanate. Pediatr Dermatol 2012;29:770-1.

E33. Kardaun SH, Tupker RA. Symmetrical drug-related intertriginous and flexural exanthema (baboon syndrome) induced by omeprazole. Int J Dermatol 2012; 51:1134-7.

E34. Peeters D, Baeck M, Dewulf V, Tennstedt D, Dachelet C. A case of SDRIFE induced by Nuvaring(®). Contact Dermatitis 2012;66:110-1.

E35. Rao A, Francis N, Morar N. Clozapine-induced symmetrical drug-related intertriginous and flexural exanthema: first reported cases. Br J Dermatol 2012; $166: 1142-3$.

E36. Ozkaya E, Babuna G. A challenging case: symmetrical drug related intertriginous and flexural exanthem, fixed drug eruption, or both? Pediatr Dermatol 2011;28:711-4.

E37. Allain-Veyrac G, Lebreton A, Collonnier C, Jolliet P. First case of symmetric drug-related intertriginous and flexural exanthema (sdrife) due to rivastigmine? Am J Clin Dermatol 2011;12:210-3.

E38. Chong W-S, Lim H-LJ. Symmetrical drug-related intertriginous and flexural exanthema (baboon syndrome) with fever induced by oral cloxacillin: does fever confound the diagnosis? J Dermatol 2010;37:830-2.

E39. Ferreira O, Mota A, Morais P, Cunha AP, Azevedo F. Symmetrical drugrelated intertriginous and flexural exanthema (SDRIFE) induced by telmisartan-hydrochlorothiazide. Cutan Ocul Toxicol 2010;29:293-5.

E40. Elmariah SB, Cheung W, Wang N, Kamino H, Pomeranz MK. Systemic drugrelated intertriginous and flexural exanthema (SDRIFE). Dermatol Online J 2009;15:3.

E41. Akay BN, Sanli H. Symmetrical drug-related intertriginous and flexural exanthem due to oral risperidone. Pediatr Dermatol 2009;26:214-6.

E42. Thierman S, Chinthraja RS. Symmetrical drug-related intertriginous and flexural exanthema after coronary artery angiography. J Hosp Med 2009;4:203.

E43. Daito J, Hanada K, Katoh N, Katoh S, Sakamoto K, Asai J, et al. Symmetrical drug-related intertriginous and flexural exanthema caused by valacyclovir. Dermatol Basel Switz 2009:218:60-2.

E44. Handisurya A, Stingl G, Wöhrl S. SDRIFE (baboon syndrome) induced by penicillin. Clin Exp Dermatol 2009;34:355-7.

E45. Diaz Ley B, Guhl G, Eguren Michelena C, Fernandez Herrera J, Fraga J, Garcia Diez A. Flexural cutaneous eruption due to palifermin. Br J Haematol 2008;140:464-5.

E46. Dhingra B, Grover C. Baboon syndrome. Indian Pediatr 2007;44:937.

E47. Arnold AW, Hausermann P, Bach S, Bircher AJ. Recurrent flexural exanthema (SDRIFE or baboon syndrome) after administration of two different iodinated radio contrast media. Dermatol Basel Switz 2007;214:89-93.

E48. Treudler R, Simon JC. Symmetric, drug-related, intertriginous, and flexural exanthema in a patient with polyvalent intolerance to corticosteroids. J Allergy Clin Immunol 2006;118:965-7. 